\title{
Time series photometry of the dwarf planet ERIS (2003 UB313) ${ }^{\star}$
}

\author{
G. Carraro ${ }^{1,2}$, M. Maris ${ }^{3}$, D. Bertin ${ }^{2}$, and M. G. Parisi ${ }^{2,4}$ \\ 1 Dipartimento di Astronomia, Università di Padova, Vicolo Osservatorio 2, 35122 Padova, Italy \\ e-mail: gcarraro@das.uchile.cl \\ 2 Departamento de Astronomía, Universidad de Chile, Casilla 36-D, Santiago, Chile \\ 3 INAF, Osservatorio Astronomico di Trieste, via Tiepolo 11, 40013 Trieste, Italy \\ ${ }^{4}$ Facultad de Ciencias Astronómicas y Geofísicas de la UNLP, IALP-CONICET, Paseo del Bosque s/n, La Plata, Argentina
}

Received 9 October 2006 / Accepted 19 October 2006

\section{ABSTRACT}

\begin{abstract}
Context. The dwarf planet Eris (2003 UB313, formerly known also as "Xena") is the largest KBO discovered up to now. Despite being larger than Pluto and having many similarities to it, it has not been possible so far to detect any significant variability in its light curve, preventing the determination of its period and axial ratio.

Aims. We attempt to assess the level of variability of the Eris light curve by determining its $B V R I$ photometry with a target accuracy of $0.03 \mathrm{mag} / \mathrm{frame}$ in $R$ and a comparable or better stability in the calibration.

Methods. Eris has been observed between November 30th and December 5th, 2005 with the Y4KCam onboard the $1.0 \mathrm{~m}$ Yale telescope at Cerro Tololo Interamerican Observatory, Chile in photometric nights.

Results. We obtain 7 measures in $B, 23$ in $V, 62$ in $R$, and 20 in $I$. Averaged $B, V$, and $I$ magnitudes as colors are in agreement within $\approx 0.03$ mag with measures from Rabinowitz et al. (2006, [arXiv:astro-ph/0605745]) taken on the same nights. Night-averaged magnitudes in $R$ show a statistically significant variability over a range of about $0.05 \pm 0.01 \mathrm{mag}$. This cannot be explained by known systematics, background objects, or some periodical variation with periods less than two days in the lightcurve. The same applies to $B, V$ and to a lesser extent to $I$, due to larger errors.

Conclusions. In analogy with Pluto and if confirmed by future observations, this "long term" variability might be ascribed to a slow rotation of Eris, with periods longer than 5 days, or to the effect of its unresolved satellite "Dysnomea", which may contribute for $\approx 0.02$ mag to the total brightness.
\end{abstract}

Key words. Kuiper Belt - solar system: general - minor planets, asteroids - techniques: photometric

\section{Introduction}

Since its discovery the dwarf planet 2003 UB313 has attracted a lot of attention, being the first Trans Neptunian Object (TNO) larger than Pluto ever discovered (Brown et al. 2005). This object, recently baptized "Eris" (IAU 2006), revealed a number of features in common with Pluto, despite being a member of the family of the scattered TNO (Sheppard 2006). As an example, like Pluto, Eris has a satellite named "Dysnomea" with an orbital period of about two weeks, a brightness of about $2 \%$ of that of Eris, and a semi-major axis of $\approx 5 \times 10^{4} \mathrm{~km}$ (Brown et al., 2006a). Eris' IR spectrum is clearly dominated by $\mathrm{CH}_{4}$ absorption bands (Brown et al., 2006b) and perhaps shows $\mathrm{N}_{2}$ bands (Licandro et al., 2006). When compared with other TNOs its colors are quite neutral and not significantly reddened (Rabinowitz et al., 2006). Its phase function at small phase angles is quite flat (Rabinowitz et al., 2006). Up to now its light curve did not reveal any trace of significant variability or periodicity (Brown et al., 2005; Rabinowitz et al., 2006). These features suggest Eris to be an icy body that is subject to frequent resurfacing, likely due to evaporation and redeposition of a tiny atmosphere as its heliocentric distance changes (Brown et al., 2005; Rabinowitz et al., 2006). In this Letter we present $B V R I$ photometry of Eris

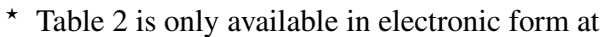
http: //www . aanda.org obtained during 5 nights in late 2005 with the aim of building a light curve and searching for possible periodicity. The same data set is used to better constrain the optical colors of the object.

\section{Observations and data reduction}

We observed Eris over 5 consecutive nights (November 30 to December 4, 2005). Photometric data were obtained with the Y4KCam CCD onboard the Yale $1.0 \mathrm{~m}$ telescope at Cerro Tololo Interamerican Observatory, which is operated by the SMARTS consortium $^{1}$. The Y4KCam instrument is a $4096 \times 4096$ CCD with a pixel scale of $0.289^{\prime \prime}$, which allows one to observe a field 20 arcmin on a side on the sky. An image of the field around Eris is shown in Fig. 1. A series of images in $B V R I$ was acquired to constraint both the light curve and the colors. A total of $7 \mathrm{im}-$ ages in B, 23 in $V, 62$ in $R$ and 20 in $I$ has been obtained over the observing run, and the exposure times were $300-600 \mathrm{~s}$. The nights were all photometric except for the last one (December 4, 2005), with typical seeing ranging from 0.8 to 1.2 arcsec. The images were cleaned and pre-reduced using the pipeline developed by Phil Massey ${ }^{2}$. To extract Eris magnitudes, we used the

\footnotetext{
http://www . astro.yale.edu/smarts/

2 http://www . lowell. edu/users/massey/obins/ y4kcamred.html
} 


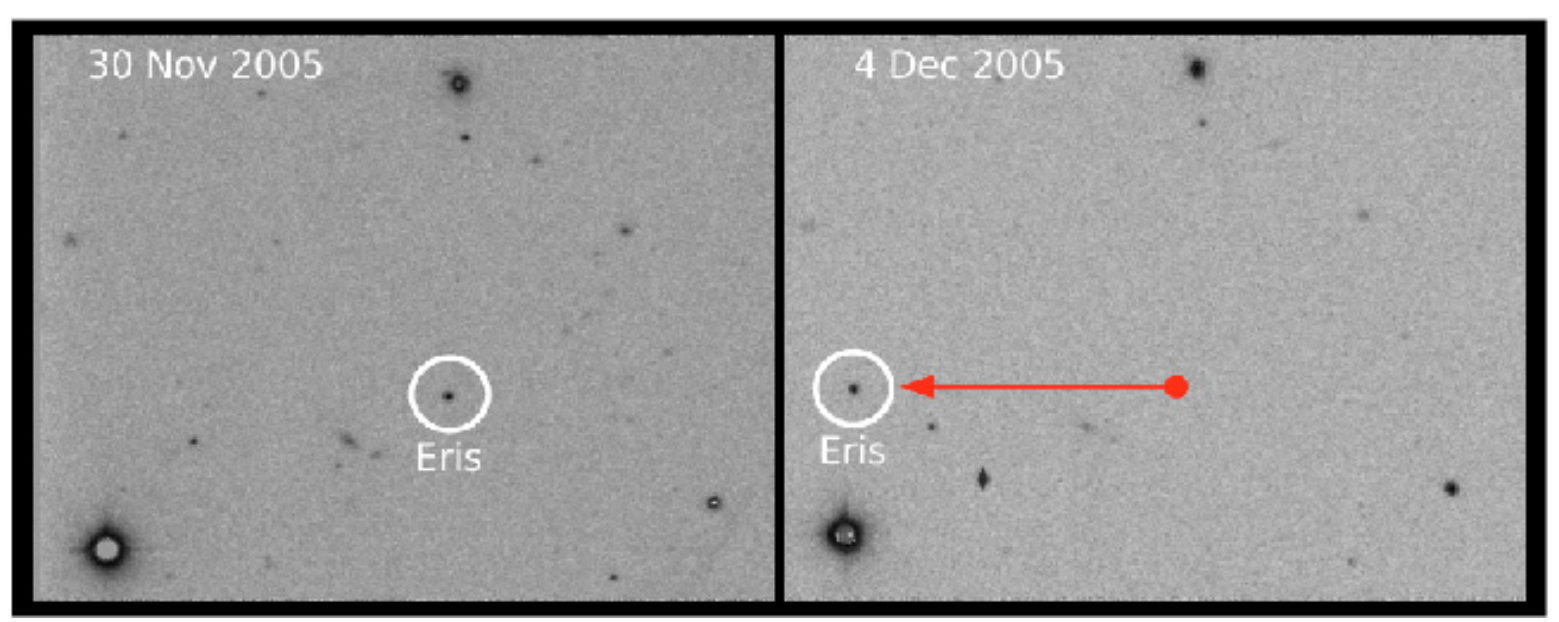

Fig. 1. Eris' position on the first (left panel) and last night (right panel). The area is $3.5 \times 2.7$ arcmin. North in down, east to the right. The arrow indicates Eris' motion over the whole run.

Table 1. Weighted averages of $B V R I$ for each night.

\begin{tabular}{cccccc}
\hline \hline Night & Date & $\begin{array}{c}B \\
{[\mathrm{mag}]}\end{array}$ & $\begin{array}{c}V \\
{[\mathrm{mag}]}\end{array}$ & $\begin{array}{c}R \\
{[\mathrm{mag}]}\end{array}$ & $\begin{array}{c}I \\
{[\mathrm{mag}]}\end{array}$ \\
\hline 1 & Nov. 30, 2005 & $19.619 \pm 0.041$ & $18.766 \pm 0.025$ & $18.384 \pm 0.010$ & $18.019 \pm 0.030$ \\
2 & Dec. 1, 2005 & $19.540 \pm 0.038$ & $18.768 \pm 0.015$ & $18.368 \pm 0.007$ & $18.029 \pm 0.032$ \\
3 & Dec. 2, 2005 & $19.651 \pm 0.066$ & $18.772 \pm 0.015$ & $18.388 \pm 0.007$ & $17.948 \pm 0.022$ \\
4 & Dec. 3, 2005 & $19.678 \pm 0.052$ & $18.788 \pm 0.016$ & $18.397 \pm 0.007$ & $17.975 \pm 0.022$ \\
5 & Dec. 4, 2005 & $19.616 \pm 0.077$ & $18.802 \pm 0.015$ & $18.422 \pm 0.007$ & $18.039 \pm 0.027$ \\
\hline
\end{tabular}

QPHOT task within IRAF $^{3}$, which allows one to measure aperture photometry. For Eris we used a small aperture (7 pixels). Together with Eris we measured 5 field stars with roughly the same magnitude $(17.53 \leq\langle R\rangle \leq 19.25)$. Due to the slow motion of Eris and the wide field covered by the CCD, we could measure the same 5 stars every night and thus tie the photometry to the same zero point for the entire dataset. For the field stars we used a larger aperture (18 pixels). Absolute magnitudes were derived by shifting Eris magnitudes to the first night using the reference field stars. A set of bright stars in the first night were used to aperture-correct the magnitudes. Aperture corrections were found to be small, of the order of 0.05-0.12 mag. The zero points of the photometry were then obtained through the observation of 50 standard stars in the Landolt (1982) fields PG0231, SA92, and Rubin149, using a large aperture of 18 pixels as well. The magnitudes were also color-corrected using Eris' mean colors. The final photometry, consisting of 115 data points, is reported in Table 2 together with the photometric error, UT time, and filter. In the same period we observed, Rabinowitz et al. (2006) obtained $2 \mathrm{~B}, 4 \mathrm{~V}$ and $2 \mathrm{I}$ images of Eris. We compared our photometry with theirs, and found a good agreement, being $\Delta B=0.05, \Delta V=-0.032$, and $\Delta I=0-0.022$, where the form is ours photometry minus theirs. We do not have direct comparison with $R$, since these authors did not publish data in $R$ for these nights.

\section{Light curve and period hunting}

Figure 2 shows night-by-night time dependencies in the $R$ band. It is evident that, even removing the four measures with anomalously large errors $(\Delta R>0.04 \mathrm{mag})$, the dispersion of data

${ }^{3}$ IRAF is distributed by the National Optical Astronomy Observatories, which are operated by the Association of Universities for Research in Astronomy, Inc., under cooperative agreement with the National Science Foundation.

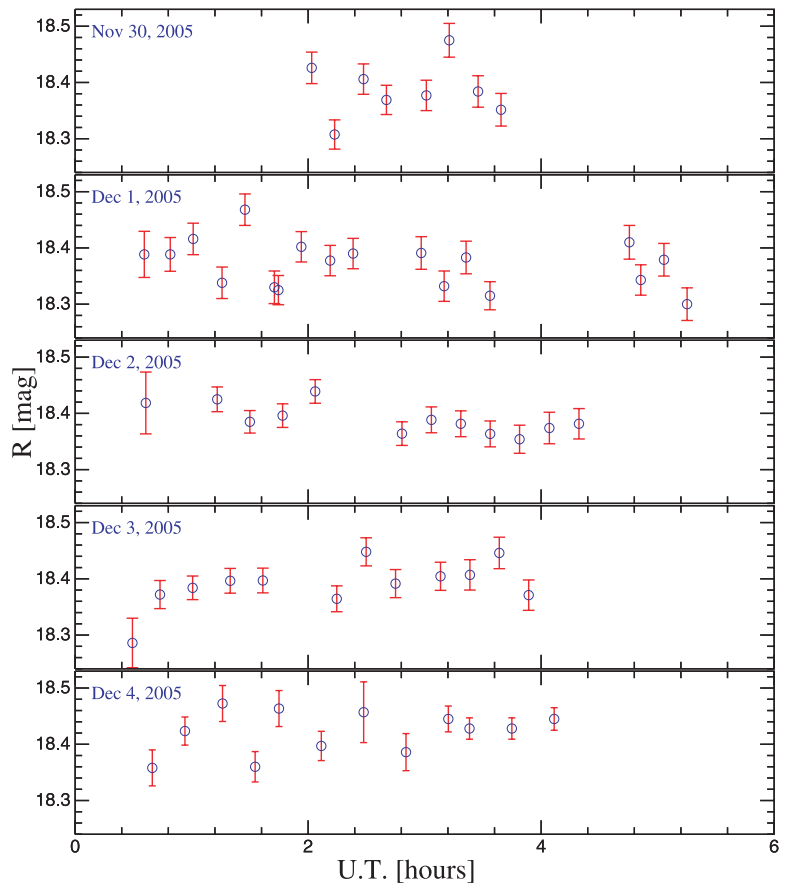

Fig. 2. $R$ light curves of Eris (2003 UB313). From the top to the bottom the light curves for Nov. 30 to Dec. 4, 2005 are shown.

cannot be attributed just to random errors. The weighted average for all of the five nights gives $\bar{R}=18.3916 \pm 0.0033 \mathrm{mag}$ with $\chi^{2}=136$ and 57 d.o.f. (degree of freedom); a $\chi^{2}$-test rejects the hypothesis of random fluctuations at a confidence level (c.l.) $\sim 2 \times 10^{-8}$, which is insensitive to the exclusion of the bad measures. Inspection of the $R$ frames shows that Eris is moving very slowly in an uncrowded field (see Fig. 1), with no evident objects in the background. Moreover, due to the short 


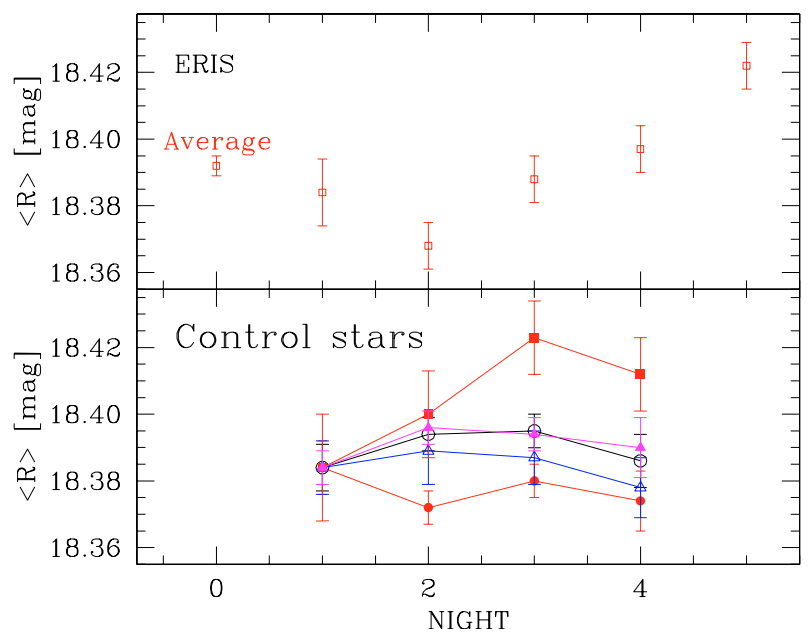

Fig. 3. Upper panel: Eris weighted averages $R$ mag as a function of night (see Table 1). The point in blue (dark gray in bw print) at night 0 is the weighted average over the 5 nights. Lower panel: weighted averages of $R$ as a function of the night for the 5 field stars discussed in the text. Only the data for the first 4 nights are plotted, since the fifth was not photometric. The magnitude of these stars has been arbitrarily shifted to the first night Eris average $R$ mag.

exposures and the low proper motion, both Eris and the field stars are round without trailing. All together this seems to exclude at least the most common systematic effects. The observing conditions (heliocentric distance $r_{\mathrm{h}}$, geocentric distance $\Delta$, as phase angles $\alpha$ ) could be responsible for the effect. However, during the five nights they were fairly stable. In fact, the object moves about 95 arcsec in 5 nights. The change in $r_{\mathrm{h}} \Delta$ explains no more than $1.7 \times 10^{-3} \mathrm{mag}$. On the other hand, the phase angle varies of $\Delta \alpha=0.0246^{\circ}$ during the observations. No phase coefficients in $R$ have been published so far for Eris, but assuming as an upper limit the same phase coefficient of $V$ in Rabinowitz et al. (2006), the phase effect would account at most for $3.5 \times 10^{-3}$ mag. In conclusion, obvious changes in the observing conditions exclude geometrical effects. Table 1 reports weighted averages for magnitudes taken in the same night, while the lower frame of Fig. 3 displays the same data for the $R$ filter. A clear trend appears in $R$ for nights 2 to 5 .

A $\chi^{2}$ test rejects the hypothesis of random fluctuations at c.l. $\approx 2 \times 10^{-6}$, inclusion of night 1 does not change this result. This is robust against selection of data according to the U.T. of observation (as evident from Fig. 2 on night 1 Eris has been observed just between U.T. $=2$ and U.T. $=4$, and considering only data in that U.T. interval does not change the result) and replacing weighted averages with median estimation of nightly centroids. The difference between nights 5 and 2 is $0.054 \pm 0.010 \mathrm{mag}$, equivalent to $5.4 \sigma$. A linear fit for nights 2 to 5 gives a slope $R^{\prime} \approx 0.0170 \pm 0.0002 \mathrm{mag} /$ day with $\chi_{\text {linear }}^{2}=0.99$ equivalent to c.l. $\approx 0.6$ that residual fluctuations are just due to errors. A parabolic fit including all the nights gives $\chi_{\text {parabolic }}^{2}=$ 2.35 equivalent to a c.l. $\approx 0.3$.

The lower panel of Fig. 3 compares variations for 5 field stars having $R$ in the approximated range $17.5 \mathrm{mag}-19.3 \mathrm{mag}$ encompassing the range of Eris $R$ magnitudes. Magnitudes are measured frame by frame and averaged over each night in the same manner as the Eris data. To highlight the variations, the first night of each series has been shifted to the averaged $R$ for Eris, $R=18.39$ mag. It is evident that field stars are stable with peak-to-peak variations in $R$ of about $0.01 \mathrm{mag}$. The only star departing from this value is the weakest in the series having $R=$ $19.3 \mathrm{mag}$. In addition, the expected random errors for field stars are similar to the random errors for Eris. Larger errors appearing for $R$ larger then 19 mag. A convincing test of the calibration stability comes from the fact that variability indicators for field stars (either peak-to-peak variation, or the rms between the 4 nights, the $\chi^{2}$ for fitting against a constant value or better the related significativity) plotted as a function of their mean magnitude are constant for $R$ up to $\approx 19$ mag. Moreover, for Eris the indicators of variability always differ significantly from the values obtained for field stars below $R=19$ mag. Peak-to-peak variation for field stars is $\approx 0.011 \mathrm{mag}-0.012 \mathrm{mag}$ v.z. Eris $0.029 \mathrm{mag}$. Night-by-night rms for the field stars is $\approx 0.005-0.006$ mag, v.z. Eris 0.012 mag. The significativity of fluctuations for field stars is always below the $60 \%$ level v.z. Eris showing fluctuations with a significativity larger than $98 \%$. In addition, field starts fluctuations are not very much correlated with Eris fluctuation; in some cases field stars are anticorrelated with Eris and correlations are not very significant. All this supports the idea that Eris brightness variations are not due to calibration errors. Looking at the other filters the same trend in nights 2 to 5 appears for $V, B$, and marginally $I$ but with a lower significance owing to larger errors. We excluded that the trend is connected to fluctuations in the zero point calibration as derived from standard stars. The nightby-night zero point for $R, R_{0}$, is spread of $\Delta R_{0} \approx 0.004 \mathrm{mag}$, consistent with its rms, $\sigma_{R_{0}} \approx 0.008 \mathrm{mag}$, and just has a marginal trend with slope $4 \times 10^{-4} \mathrm{mag} / \mathrm{day}$, to be compared with the spread of Eris over the first four nights of $\Delta R_{\text {Eris }}=0.029$ mag. For $V, \Delta V_{0} \pm \sigma_{V_{0}} \approx 0.005 \pm 0.01$ mag to be compared with $\Delta V_{\text {Eris }}=0.022 \mathrm{mag}$. Besides, $V$ and $R$ are correlated by the correlation coefficient $\rho_{V R}=0.92$. At the same time, $V-R$ computed night-by-night is fairly stable. A fit against the case of constant $V-R$ has $\chi^{2}=0.85$ corresponding to a c.l. $\approx 0.93$ that fluctuations about the averaged value $(V-R=0.388 \pm 0.008$ from Table. 1) are just due to chance. As a comparison, the $V_{0}-R_{0}$ on a night-by-night basis has an $\mathrm{rms}=0.006$ mag with a c.l. $\approx 0.99$ for random fluctuations. Correlation between colors in light curves is expected if Eris is an icy body frequently resurfaced by atmospheric freezing. In this case a uniform layer of frozen gasses should hide color variations.

$B$ and $I$ have less precise calibration and random errors and sparser coverages, but for completeness, it is worthwhile to extend the discussion to these data, too. $B$ and $I$ are less correlated with $R$ having respectively $\rho_{\mathrm{BR}}=0.465$, and $\rho_{\mathrm{IR}}=0.137$. The correlation between $B$ and $R$ is very sensitive to the exclusion of the last night. Then for the first four nights $\rho_{\mathrm{BR}}=0.995$, In addition the c.l. against random fluctuations are just 0.23 and 0.04 respectively for $B$ and $I$. Again, the variability in $B$ and $I$ cannot be reconciled with variations in $B_{0}$ and $I_{0}$, since $\Delta B_{\text {Eris }}=0.138 \mathrm{mag}$ and $\Delta I_{\text {Eris }}=0.081 \mathrm{mag}$, while $\Delta B_{0} \pm \sigma_{B_{0}} \approx 0.03 \pm 0.01 \mathrm{mag}$ and $\Delta I_{0} \pm \sigma_{I_{0}} \approx 0.039 \pm 0.018 \mathrm{mag}$. Note the different behavior of $I$ in the second night. While $B, V$, and $R$ have lower or equal magnitudes on night two respect to night one and three, $I$ shows the opposite trend. Indeed, after removing the second night, $\rho_{\mathrm{IR}}=0.57$, while removing even the first, $\rho_{\mathrm{IR}}=0.998$.

If this tiny time dependence is not due to some unaccounted problem in the data, would this be a sign of an aliasing with short term variability? We attempt to assess whether the dispersion in the data can be ascribed to some periodical variations in the light curve over periods shorter than 5 nights. The phase dispersion minimization method applied to data binned in chunks of $1 \mathrm{~h}$ favors periodicity of about $30 \mathrm{~h}$. Other possible periods are much more sensitive to the details of the method, as the number of phase bin or the step in periods. An inspection 
of data plotted as a function of phase for a $30 \mathrm{~h}$ periods suggests a scattered, non sinusoidal light curve, with a maximum peak-to-peak variation of $\approx 0.06$ mag and a single maximum, but the fitting is marginal $\left(\chi^{2}=116.74\right.$ with c.l. $=2 \times 10^{-5}$ that deviations from the fit are not just due to random errors). The periodogram of data does not allow us to identify any noticeable periodicity between 1 and $100 \mathrm{~h}$. This is true even after the exclusion of periods heavily affected by aliasing $(6 \mathrm{~h}, 8 \mathrm{~h}$, $12 \mathrm{~h}, 24 \mathrm{~h}$, and $48 \mathrm{~h}$ ). In particular, the $30 \mathrm{~h}$ period is just outside the $24 \mathrm{~h}$ side-lobe, and the improvement in the $\chi^{2}$ for fitting data with an $\approx 30 \mathrm{~h}$ period is again marginal. To have a more robust test we play numerical experiments with simulated sinusoidal signals plus noise. Here we consider periods in the range $1-100 \mathrm{~h}$, amplitudes $\leq 0.05 \mathrm{mag}$, constant $R$ mag in the $\pm 5 \sigma$ of our data, and phases in the range $0-2 \pi$. Simulated data has been re-binned on a night-by-night basis and compared to night averaged data computing the corresponding $\chi_{\sin }^{2}$. As a comparison we take $\chi_{\text {sin }}^{2}$ with $\chi_{\text {const }}^{2}, \chi_{\text {linear }}^{2}=2.36$ (computed over 5 nights), and $\chi_{\text {parabolic }}^{2}$ as defined before. Our results show no significant improvement in the fit by assuming a sinusoidal signal in the data. In at most $3 \%$ of our $3 \times 10^{5}$ simulated realizations, we obtained $\chi_{\text {sin }}^{2}<\chi_{\text {const }}^{2}$. The fraction drops to $0.05 \%$ and $0.003 \%$, respectively for $\chi_{\text {sin }}^{2}<\chi_{\text {linear }}^{2}$ and $\chi_{\text {sin }}^{2}<\chi_{\text {parabolic }}^{2}$. To have an extreme case of non-sinusoidal signal we also consider the case of a square wave with variable amplitude, period, phase and dutycycle obtaining a largely worst fit. In conclusion the long term variability in our data ca not be explained by the aliasing of an under-sampled short-term variability.

\section{Colors}

We computed weighted mean colors of Eris. These are derived from the weighted mean of all the measures in each filter. We obtain $B-V=0.823 \pm 0.023, V-R=0.391 \pm 0.023, R-I=$ $0.386 \pm 0.012$, and $V-I=0.777 \pm 0.013$, quite in agreement with Rabinowitz et al. (2006, Table 4). Following the same vein of the discussion in this paper, we confirm that the colors of ERIS are solar, with only $B-V$ being marginally redder than the Sun Hainaut \& Delsanti (2002). These colors corroborate the idea that Eris is an icy body.

\section{Discussion and conclusions}

We have presented time series photometry in BVRI pass-bands of the dwarf planet Eris (2003 UB313).

Looking at the data we have presented and analyzed (in particular $V$ and $R$ ), it is possible to say that some genuine time variability is present with a reasonable level of confidence. If this will be confirmed by further observations it would indicate a light curve with a long term variability. Likely, one with periods greater than 5 days and amplitudes $\approx 0.05 \mathrm{mag}$. Such a small amplitude would indicate a low axial ratio for Eris or that this body is seen nearly pole-on from the Earth. In the first case Eris would be more symmetric in shape than other known KBOs or Pluto itself. In the second case, due to the large distance to the Sun, Eris is pole-on with respect to the Sun, too. Presently Eris is near its aphelion and if it has an axial ratio comparable to that of Pluto, we should expect that the maximum amplitude of its light curve would be observed toward the epoch in which it will have an anomaly of $\approx 90^{\circ}$. However, even a change of $10^{\circ}$ in its orientation would produce a significant increment in the amplitude of its light curve.

Interestingly enough for the evolution of resurfacing is the fact that in case Eris was seen pole-on at aphelion, it would have to be pole-on even at perihelion. Having a so large orbital ellipticity, the solar irradiation at aphelion would be 6.6 times smaller than the irradiation at perihelion. Depending on the details of such resurfacing mechanism and atmospheric circulation, it would not be a surprise to discover significant differences between the two hemispheres of Eris. As an example, one can speculate that the region of the aphelion pole would be more rich in volatiles than the opposite region. If so, even the spectroscopic signature of the Eris surface will have to show secular variations correlated with the light curve amplitude.

Finally, in trying to understand Eris' light curve, one cannot neglect that the presence of the un-resolved satellite could distort it. Dysnomea indeed may contribute for up to $\approx 0.02 \mathrm{mag}$ to the time variability of brightness with an expected orbital period of 2 weeks. However, even assuming that the line of nodes of the orbit of the satellite is oriented toward the Sun, an eclipse or a transit would last for about one tenth of day, compatible with the time scale of our observations over each night. Howcver an eclipse or a transit would cause a drop in brightness, while our data suggest rather the opposite behavior. In addition, an eclipse or a transit would affect only one night and not the subsequent ones, due to the small phase angle with which we are observing the system.

Acknowledgements. The work of G.C. was supported by Fundacion Andes. The work of M.M. was partially supported by INAF FFO for free research 2006 (Fondo Ricerca Libera). The authors acknowledge the referee, David Rabinowitz, for useful suggestions.

\section{References}

Brown, M. E., Trujillo, C. A., \& Rabinowitz, D. L. 2005, ApJ, 635, L97 Brown, M. E., van Dam, M. A., Bouchez, A. H., et al. 2006a, ApJ, 639, L43 Brown, M. E., Schaller, E. L., Roe, H. G., Rabinowitz, D. L., \& Trujillo, C. A. 2006b, preprint

Hainault, O. R., \& Delsanti, A. C. 2002, A\&A, 389, 641

IAU 2006, IAU resolution 5A, 26th IAU General Assembly, Prague 2006

Licandro, J., Grundy, W. M., Pinilla-Alonso, N., \& Leisy, P. 2006, A\&A, 458, L5

Sheppard, S. S. 2006, Nature, 439, 541

Rabinowitz, D. L., Schaefer, B. E., \& Tourtellotte, S. W. 2006, [arXiv: astro-ph/0605745] 
G. Carraro et al.: Time series photometry of the dwarf planet ERIS (2003 UB313), Online Material p 1

\section{Online Material}


G. Carraro et al.: Time series photometry of the dwarf planet ERIS (2003 UB313), Online Material p 2

Table 2. Log of observations.

\begin{tabular}{|c|c|c|}
\hline$\overline{\overline{\text { UT }}}$ & Filter & Magnitude \\
\hline \multicolumn{3}{|c|}{ 2005, Nov. 30} \\
\hline 01:53:55 & $I$ & $18.058 \pm 0.050$ \\
\hline 02:01:50 & $R$ & $18.426 \pm 0.028$ \\
\hline 02:07:44 & V & $18.755 \pm 0.033$ \\
\hline $02: 13: 38$ & $R$ & $18.308 \pm 0.026$ \\
\hline 02:19:35 & $B$ & $19.557 \pm 0.054$ \\
\hline $02: 28: 31$ & $R$ & $18.406 \pm 0.027$ \\
\hline $02: 34: 25$ & I & $18.021 \pm 0.055$ \\
\hline 02:40:19 & $R$ & $18.369 \pm 0.026$ \\
\hline 03:00:52 & $R$ & $18.377 \pm 0.027$ \\
\hline 03:06:40 & V & $18.779 \pm 0.038$ \\
\hline $03: 12: 41$ & $R$ & $18.475 \pm 0.030$ \\
\hline 03:18:37 & $B$ & $19.703 \pm 0.063$ \\
\hline $03: 27: 32$ & $R$ & $18.384 \pm 0.028$ \\
\hline $03: 33: 26$ & $I$ & $17.974 \pm 0.053$ \\
\hline 03:39:20 & $R$ & $18.352 \pm 0.029$ \\
\hline \multicolumn{3}{|c|}{ 2005, Dec. 01} \\
\hline $00: 35: 37$ & $R$ & $18.389 \pm 0.041$ \\
\hline $00: 41: 31$ & $V$ & $18.829 \pm 0.042$ \\
\hline 00:48:59 & $R$ & $18.389 \pm 0.030$ \\
\hline $00: 54: 53$ & V & $18.841 \pm 0.042$ \\
\hline 01:00:48 & $R$ & $18.416 \pm 0.028$ \\
\hline 01:06:43 & $B$ & $19.557 \pm 0.055$ \\
\hline 01:15:39 & $R$ & $18.338 \pm 0.028$ \\
\hline 01:21:34 & I & $17.967 \pm 0.052$ \\
\hline $01: 27: 28$ & $R$ & $18.468 \pm 0.028$ \\
\hline 01:42:37 & $R$ & $18.330 \pm 0.029$ \\
\hline 01:44:40 & $R$ & $18.325 \pm 0.026$ \\
\hline 01:50:35 & V & $18.701 \pm 0.033$ \\
\hline 01:56:29 & $R$ & $18.402 \pm 0.027$ \\
\hline $02: 02: 25$ & $B$ & $19.526 \pm 0.051$ \\
\hline $02: 11: 21$ & $R$ & $18.378 \pm 0.027$ \\
\hline $02: 17: 15$ & I & $18.237 \pm 0.067$ \\
\hline 02:23:09 & $R$ & $18.390 \pm 0.027$ \\
\hline $02: 58: 14$ & $R$ & $18.391 \pm 0.029$ \\
\hline 03:04:09 & V & $18.779 \pm 0.039$ \\
\hline 03:10:03 & $R$ & $18.332 \pm 0.027$ \\
\hline $03: 15: 57$ & I & $18.038 \pm 0.054$ \\
\hline $03: 21: 21$ & $R$ & $18.383 \pm 0.029$ \\
\hline $03: 27: 45$ & V & $18.807 \pm 0.039$ \\
\hline 03:33:39 & $R$ & $18.315 \pm 0.025$ \\
\hline 03:39:33 & I & $18.104 \pm 0.062$ \\
\hline $03: 57: 17$ & I & $18.238 \pm 0.069$ \\
\hline 04:09:13 & V & $18.708 \pm 0.035$ \\
\hline $04: 45: 27$ & $R$ & $18.410 \pm 0.030$ \\
\hline $04: 51: 18$ & $R$ & $18.343 \pm 0.027$ \\
\hline $05: 03: 15$ & $R$ & $18.379 \pm 0.029$ \\
\hline $05: 15: 10$ & $R$ & $18.300 \pm 0.029$ \\
\hline \multicolumn{3}{|c|}{ 2005, Dec. 02} \\
\hline 00:28:00 & V & $18.818 \pm 0.063$ \\
\hline $00: 36: 24$ & $R$ & $18.419 \pm 0.055$ \\
\hline 01:13:11 & $R$ & $18.425 \pm 0.022$ \\
\hline 01:21:35 & V & $18.805 \pm 0.028$ \\
\hline 01:29:59 & $R$ & $18.385 \pm 0.020$ \\
\hline 01:38:25 & $B$ & $19.651 \pm 0.066$ \\
\hline 01:46:50 & $R$ & $18.396 \pm 0.021$ \\
\hline 01:55:14 & I & $17.914 \pm 0.036$ \\
\hline 02:03:38 & $R$ & $18.439 \pm 0.021$ \\
\hline $02: 48: 20$ & $R$ & $18.364 \pm 0.021$ \\
\hline $02: 55: 54$ & V & $18.722 \pm 0.028$ \\
\hline
\end{tabular}

Table 2. continued.

\begin{tabular}{|c|c|c|}
\hline$\overline{\mathrm{UT}}$ & $\overline{\text { Filter }}$ & $\overline{\text { Magnitude }}$ \\
\hline $03: 03: 28$ & $R$ & $18.389 \pm 0.023$ \\
\hline 03:11:03 & $I$ & $17.911 \pm 0.042$ \\
\hline 03:18:37 & $R$ & $18.381 \pm 0.023$ \\
\hline 03:26:11 & $V$ & $18.824 \pm 0.032$ \\
\hline 03:33:45 & $R$ & $18.363 \pm 0.023$ \\
\hline 03:41:19 & $I$ & $18.001 \pm 0.047$ \\
\hline 03:48:53 & $R$ & $18.354 \pm 0.025$ \\
\hline 04:04:14 & $R$ & $18.374 \pm 0.028$ \\
\hline 04:11:52 & $I$ & $18.008 \pm 0.052$ \\
\hline 04:19:29 & $R$ & $18.381 \pm 0.027$ \\
\hline 04:56:35 & $V$ & $18.742 \pm 0.032$ \\
\hline \multicolumn{3}{|c|}{ 2005, Dec. 03} \\
\hline $00: 21: 06$ & $V$ & $18.719 \pm 0.094$ \\
\hline $00: 29: 30$ & $R$ & $18.286 \pm 0.044$ \\
\hline $00: 43: 42$ & $R$ & $18.372 \pm 0.025$ \\
\hline 00:52:06 & $V$ & $18.746 \pm 0.026$ \\
\hline 01:00:30 & $R$ & $18.384 \pm 0.021$ \\
\hline 01:08:57 & $B$ & $19.677 \pm 0.052$ \\
\hline 01:19:52 & $R$ & $18.396 \pm 0.022$ \\
\hline $01: 28: 16$ & $I$ & $17.905 \pm 0.036$ \\
\hline 01:36:40 & $R$ & $18.397 \pm 0.022$ \\
\hline $02: 14: 44$ & $R$ & $18.364 \pm 0.023$ \\
\hline $02: 22: 19$ & $V$ & $18.826 \pm 0.034$ \\
\hline 02:29:52 & $R$ & $18.448 \pm 0.025$ \\
\hline $02: 37: 27$ & $I$ & $17.974 \pm 0.042$ \\
\hline 02:45:01 & $R$ & $18.392 \pm 0.025$ \\
\hline 03:08:11 & $R$ & $18.404 \pm 0.025$ \\
\hline $03: 15: 45$ & $V$ & $18.820 \pm 0.034$ \\
\hline 03:23:19 & $R$ & $18.407 \pm 0.027$ \\
\hline 03:30:54 & $I$ & $18.046 \pm 0.053$ \\
\hline $03: 38: 27$ & $R$ & $18.446 \pm 0.028$ \\
\hline 03:46:01 & $V$ & $18.789 \pm 0.033$ \\
\hline 03:53:35 & $R$ & $18.371 \pm 0.027$ \\
\hline 04:01:10 & $I$ & $18.060 \pm 0.054$ \\
\hline \multicolumn{3}{|c|}{ 2005, Dec. 04} \\
\hline $00: 27: 04$ & $V$ & $18.871 \pm 0.070$ \\
\hline 00:39:44 & $R$ & $18.358 \pm 0.032$ \\
\hline 00:48:08 & $V$ & $18.825 \pm 0.031$ \\
\hline $00: 56: 33$ & $R$ & $18.424 \pm 0.025$ \\
\hline 01:04:59 & $B$ & $19.616 \pm 0.077$ \\
\hline 01:15:55 & $R$ & $18.473 \pm 0.032$ \\
\hline 01:24:19 & $I$ & $17.964 \pm 0.061$ \\
\hline 01:32:42 & $R$ & $18.360 \pm 0.027$ \\
\hline 01:44:59 & $R$ & $18.463 \pm 0.032$ \\
\hline 01:55:53 & $V$ & $18.815 \pm 0.036$ \\
\hline 02:06:47 & $R$ & $18.397 \pm 0.026$ \\
\hline 02:17:41 & $I$ & $18.036 \pm 0.077$ \\
\hline $02: 28: 36$ & $R$ & $18.457 \pm 0.054$ \\
\hline 02:39:30 & $V$ & $18.810 \pm 0.046$ \\
\hline $02: 50: 24$ & $R$ & $18.386 \pm 0.033$ \\
\hline 03:01:18 & $I$ & $18.078 \pm 0.053$ \\
\hline 03:12:12 & $R$ & $18.445 \pm 0.023$ \\
\hline 03:23:06 & $R$ & $18.428 \pm 0.019$ \\
\hline 03:34:01 & $V$ & $18.782 \pm 0.023$ \\
\hline 03:44:55 & $R$ & $18.428 \pm 0.019$ \\
\hline 03:55:49 & $I$ & $18.047 \pm 0.037$ \\
\hline 04:06:43 & $R$ & \pm 0.020 \\
\hline
\end{tabular}

